Acta Crystallographica Section A

Foundations and

Advances

ISSN 2053-2733

\section{Reflections on the magnetic pair distribution function}

\author{
William Ratcliff II
}

NIST Center for Neutron Research, NIST, Gaithersburg, MD 20899, United States

Magnets surround us. They keep reminders pinned onto our refrigerators, store data on our hard drives, are used in the motors of our cars and encode information on our credit cards. While we have made a lot of progress in determining how magnetic moments order in materials, the materials we are interested in have become increasingly complex. For many of these new materials, it is critical to understand the ordering pattern of spins at the nanoscale level. The paper by Frandsen et al. (2014) introduces a new way to observe the ordering of magnetic moments, even when the order is only on the local scale.

In traditional crystallography, we are able to use diffraction to determine the average structure of ordered materials according to the overall lattice symmetry. Given the constraints based on this symmetry, we have been able to determine the positions and species of atoms to an exquisite degree of accuracy. However, in many modern materials, there are deviations from the average symmetry and the properties of these complex materials depend critically upon their local structure. Many modern materials start from a parent compound and have their properties tailored through modifications such as chemical dopants, where the localized deviations from the long-range average structure (microstructure) critically determine the desired properties. The total scattering method was developed to address this (Toby \& Egami, 1992; Dmowski et al., 1988; Egami \& Billinge, 2013). In this technique, the atomic pair distribution function (PDF) is determined from a measurement that includes both Bragg and diffuse scattering. The Fourier transform of the data gives a measure of the real-space correlations in the material; in particular, it is a histogram of the pairwise atomic separation distances in the material. The use of the PDF has become more mainstream and has been critical in elucidating the basic physics in several systems. Beyond this, the PDF technique has been useful in describing the physics of materials ranging from high- $T_{\mathrm{c}}$ superconductors to colossal magnetoresistive materials.

However, until now, the PDF approach has not been systematically extended to magnetic systems. In this issue, Frandsen et al. (2014) extend the total scattering technique to magnetic materials, using neutrons as a probe. In their article, they derive the seminal equations that describe the magnetic pair distribution function (mPDF) and calculate it for several example systems. The mPDF consists of one term, reminiscent of the atomic PDF, which measures the pairwise correlations of the projection of the moments normal to the separation axis between a given pair of moments. However, there is an entirely new term, which is linear in $r$ and modulated by the local spin correlations. The authors calculate the mPDF for several example systems and show that it is sensitive to the orientation of the magnetic moments with respect to the local crystallographic axes. However, this information is lost with the orientational averaging present in powders. Luckily, the technique is still rather sensitive to the nature of the coupling. In their examples, the difference between the mPDF for ferromagnetic and antiferromagnetic coupling is striking even after the powder average.
Figure 1

A cartoon of how changes in the local crystal structure can lead to changes in the local magnetic structure. In this cartoon, the substitution of a 'green' atom for a red atom leads to a local change in the orientation of a magnetic moment. 
In the future, there are several systems where the technique is likely to prove fruitful. The ability to determine how the local magnetic structure changes in response to local changes in the crystal structure is quite exciting (see Fig. 1). Multiferroics are materials in which both a ferroelectric and magnetic order parameter are present. $\mathrm{BiFeO}_{3}$ is a multiferroic at room temperature and has been the subject of intense study. This material is a G-type antiferromagnet (in which the spins alternate along the [111] direction) with a long-wavelength modulation imposed (Sosnowska et al., 1982). For device applications, it would be useful to have a ferromagnetic component that could be controlled by electric field. Theory predicts that the preferred direction of moments in this compound is strongly dependent upon the $A$-site ion (Weingart et al., 2012). It also predicts that a weak ferromagnetic component should be present and could give rise to a net moment if the modulation were suppressed (otherwise, it cancels out when averaged over the material). Doping the system with La does indeed suppress the modulation (Sosnowska et al., 1993). Magnetic PDF measurements could reveal how the local magnetic structure changes when the local environment changes. Another system of interest is the $\mathrm{Y}_{2} \mathrm{Mo}_{2} \mathrm{O}_{7}$ pyrochlore lattice. In this material, Mo spins lie on a network of corner-sharing tetrahedra. Nearest-neighbor spins are coupled antiferromagnetically. However, the geometry of the lattice does not allow the spins to order with a low-energy configuration and we would expect the material to be a spin liquid. The strength of the interactions should depend on the distance between the moments. Standard crystallographic measurements indicated no disorder. However, magnetic measurements of the material showed glassy rather than spin-liquid behavior. The mystery was solved through X-ray absorption fine structure (XAFS) analysis, which revealed that locally, the system is disordered (Booth $e t$ al., 2000). That is, there are slight differences in the bond lengths, which give rise to different strengths of interactions within the material. This is what gives rise to the glassy behavior. It would be intriguing to apply an mPDF analysis to this system to see how the local disorder changes the local magnetic structure. The technique is also likely to prove powerful in the case of dilute magnetic semiconductors, where it will be interesting to see how the magnetic structure changes in the vicinity of impurities. The mPDF technique should have a promising future in illuminating the local magnetic structure of complex materials.

\section{References}

Booth, C. H., Gardner, J. S., Kwei, G. H., Heffner, R. H., Bridges, F. \& Subramanian, M. A. (2000). Phys. Rev. B, 62, R755-R758.

Dmowski, W., Toby, B. H., Egami, T., Subramanian, M. A., Gopalakrishnan, J. \& Sleight, A. W. (1988). Phys. Rev. Lett. 61, 2608-2611.

Egami, T. \& Billinge, S. J. L. (2013). Underneath the Bragg Peaks: Structural Analysis of Complex Materials. Oxford, Amsterdam, San Diego: Elsevier.

Frandsen, B. A., Yang, X. \& Billinge, S. J. L. (2014). Acta Cryst. A70, 3-11.

Sosnowska, I., Loewenhaupt, M., David, W. I. F. \& Ibberson, R. M. (1993). Mater. Sci. Forum, 133-136, 683.

Sosnowska, I., Neumaier, T. P. \& Steichele, E. (1982). J. Phys. C: Solid State Phys. 15, 4835-4846.

Toby, B. H. \& Egami, T. (1992). Acta Cryst. A48, 336-346.

Weingart, C., Spaldin, N. A. \& Bousquet, E. (2012). Phys. Rev. B, 86, 094413. 